\title{
Reliability of MRI findings for Symptomatic Extraforaminal Disc Herniation in Lumbar Spine
}

\author{
Kyu Pill Moon, Kuen Tak Suh, Jung Sub Lee \\ Department of Orthopedic Surgery, Busan National University School of Medicine, Busan, Korea
}

Study Design: A retrospective study

Purpose: This study examined the reliability of the MRI findings in detecting symptomatic extraforaminal disc herniation in the lumbar spine.

Overview of Literature: There are no reports of the characteristics and reliable MRI findings of extraforaminal disc herniation.

Methods: Thirty age-and gender-matched asymptomatic volunteers and 30 patients with symptomatic extraforaminal disc herniation, who underwent surgery between March 2006 and Dec 2008, were enrolled in this study. All subjects underwent spinal MRI. The following parameters were evaluated: the presence or absence of focal eccentricity of the disc, change in the diameter of the nerve root, and displacement of the nerve root at the extraforaminal zones. Radiologic studies were reviewed blindly and independently by 3 spine surgeons.

Results: The overall agreement in determining the presence or absence of a symptomatic extraforaminal disc herniation between the three reviewers was $89.4 \%$ (161/180). The consensus showed focal eccentricity of the disc in 33 cases (55\%), a change in diameter in the nerve root in 31 cases (51.7\%), and a displacement of the nerve root in 23 cases (38.3\%). An assessment of the paired intraobserver and interobserver reliability revealed mean Kappa statistics of 0.833 and 0.667 for focal eccentricity of the disc, 0.656 and 0.556 for a change in the diameter of the nerve root, and 0.669 and 0.020 for a dis placement of the nerve root, respectively.

Conclusions: There are three possible MRI findings that can be used to determine the presence or absence of symptomatic extraforaminal disc herniation. Among these MRI findings, focal eccentricity of the disc was found to be the most reliable.

Key Words: Extraforaminal, Disc herniation, Lumbar spine, Magnetic resonance imaging, Reliability

\section{Introduction}

Extraforaminal disc herniations are uncommon causes of lumbar radiculopathy, and occur less frequently than posterior or posterolateral disc herniation at the lumbar level ${ }^{1,2}$. These types of herniation are responsible for nerve root compression at the level of or beyond the dorsal ganglion, where the root enters into or emerges from the foraminal canal $^{3}$. Assessing the precise position of the disc herniation within or beyond the foramen is important for surgeons who must choose the appropriate surgical approach ${ }^{4}$. However, there are difficulties in making a correct diagnosis before surgery because careful observations of the foraminal or extraforaminal zone are often lacking and the extent of scanning on the sagittal plane and slice thickness of the axial plane is limited. Moreover, a coexisting intraspinal pathology, spinal canal stenosis and/or disc herniation can obscure a diagnosis of far lateral entrapment of the lumbar spinal nerve ${ }^{2}$. Although $\mathrm{CT}$ and magnetic resonance imaging

Received Apr 21, 2009; 1st revised May 11, 2009; accepted May 11, 2009

Corresponding author: Jung Sub Lee, MD

Department of Orthopedic Surgery, Busan National University School of Medicine

1-10 Ami-dong, Seo-gu, Busan, 602-739, Korea

Tel: +82-51-240-7248 Fax: +82-51-247-8395, E-mail: jungsublee@pusan.ac.kr

This work was supported for two years by Pusan National University Research Grant. 
(MRI) allow successful demonstration of protrusions of the lateral disc, which account for between $6 \%$ and $10 \%$ of all lumbar disc herniations, prolapse of a lumbar disc in the far lateral zone might be overlooked ${ }^{5-8}$. The extraforaminal zone is generally not focused on in daily practice with spine MRI, particularly in the condition of large extrusion or protrusion causing descending nerve root compression or severe spinal stenosis at other levels. In particular, it is difficult to observe the disc morphology at the L5-S1 level due to the overlapping bony structures, such as the sacral ala or iliac bone, and severe decrease in disc height by degeneration.

However, there are no reports showing the characteristics and reliable MRI findings of extraforaminal disc herniation. Although extraforaminal disc herniation is important clinically, it tends to be overlooked. Therefore, this study evaluated the reliability of the MRI findings to detect symptomatic extraforaminal disc herniation in the lumbar spine.

\section{Materials and Methods}

\section{Patients}

Thirty age- and gender- matched asymptomatic volunteers and 30 patients with symptomatic extraforaminal disc herniation, who underwent surgery between March 2006 and Dec 2008, were enrolled in this study. The Institutional Review Board approved this study and informed consent was obtained from the volunteers. Patients with purely foraminal or posterolateral disc herniation were excluded because they could be diagnosed more easily through the axial and sagittal planes than those with extraforaminal disc herniation, and the main focus of this study was on extraforaminal disc herniation. All patients underwent spine MR imaging at our institution prior to surgery. The patients presented with symptoms of unilateral motor weakness and/or unilateral radiating pain in the thigh and/or leg with the exception of one who complained only of lower back pain. The symptoms disappeared after surgery in all patients.

\section{MR imaging protocol}

MR imaging was performed on a $1.5 \mathrm{~T}$ magnet (Magnetom Vision; Siemens, Erlangen, Germany) with spine coils. All axial images were obtained in the direction parallel to the lumbar discs referring to the sagittal images. Sagittal scanning was performed within the range of the vertebral body width. The T1- and T2-weighted images in axial and sagittal planes were obtained from all patients.

\section{Analysis of MR images}

Three experienced spine surgeons analyzed all MR images retrospectively independently and in consensus, and were blinded to the surgical results or clinical information. All MRIs were analyzed by the observers on different occasions with a minimum interval of 1 week. Based on previous reports, the foraminal zone was defined as the space between the medial and lateral borders of the pedicle, and the extraforaminal zone was defined as the space outside the lateral border of the pedicle ${ }^{3.9}$. Therefore, for an assessment of the nerve root status on one slice of the axial images, the nerve root located in the foraminal zone was defined as a nerve root ganglion, and the nerve root located in the extraforaminal zone was defined as an exiting nerve root. Disc herniation was determined regardless of protrusion, extrusion, or sequestrum when there was focal eccentricity in the disc contour. Extraforaminal disc herniation was diagnosed when the disc herniation was located beyond the lateral border of the pedicle.

The spine MR images were assessed as follows (Figs. 13). First, the presence or absence of eccentricity of the disc

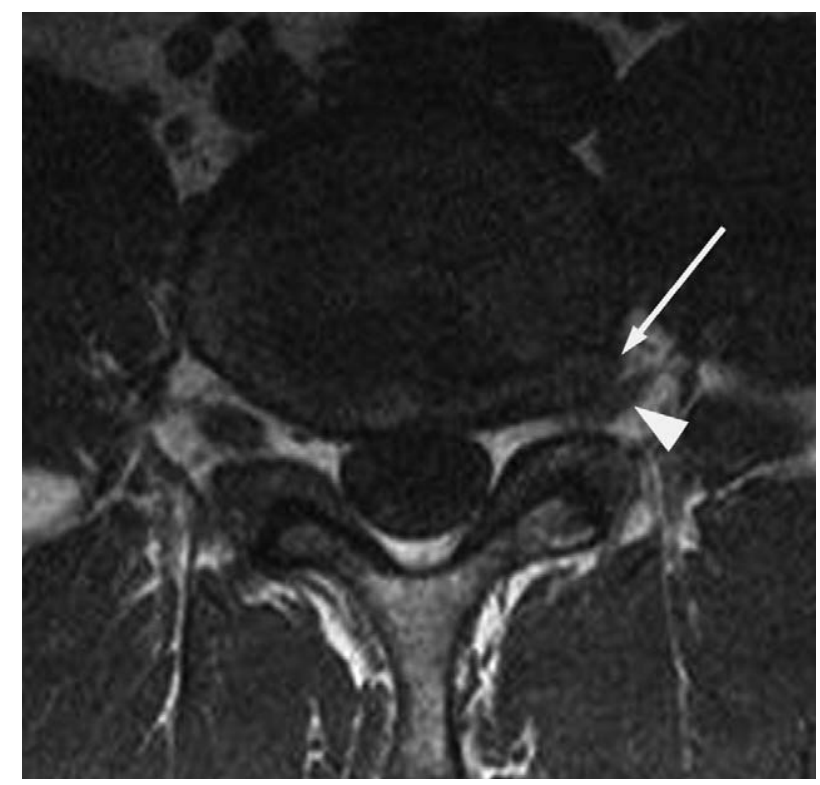

Fig. 1. Thirty-year-old man with left extraforaminal disc herniation at the L3-4 level. Axial T1-weighted image of the disc level shows obvious focal eccentricity of the disc contour (arrow) in the left extraforaminal zone. The left exiting L3 nerve root (arrowhead) was compressed due to protruded disc material. 


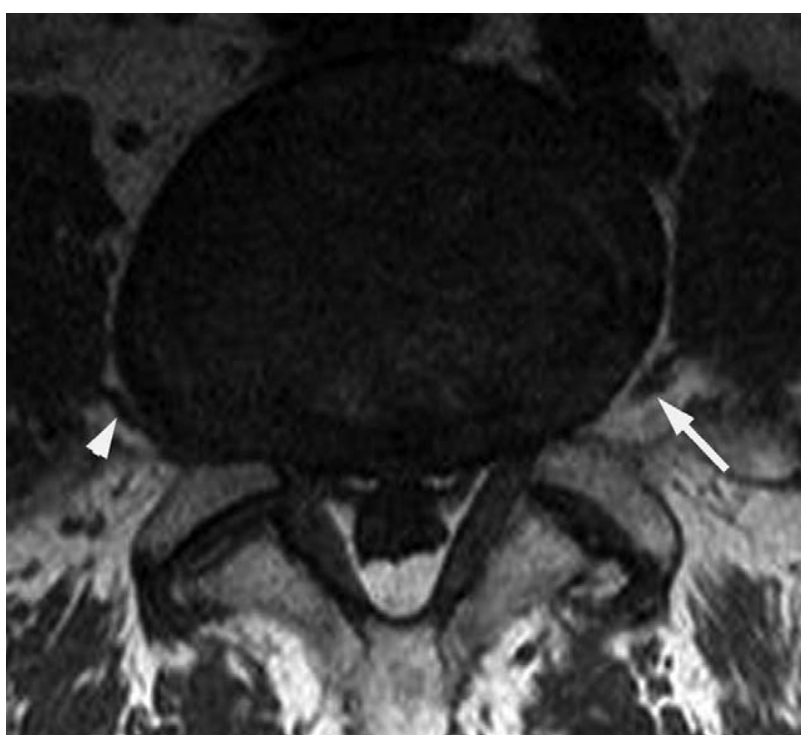

Fig. 2. Sixty-five-year-old man with right foraminal and extraforaminal disc herniation at the L5-S1 level. Focal eccentricity or obvious asymmetry of the disc contour was indistinct on the axial T1-weighted image. The right exiting L5 nerve root (arrowhead) was compressed and displaced compared to the contralateral one (arrow).

contour at the extraforaminal zone was evaluated. Eccentricity was defined as obvious focal disc extension beyond the boundary of circumferential disc around the vertebral end plates. This included protrusion, extrusion and sequestrum of the discs. Second, this study evaluated the presence or absence of any changes in the diameter of the affected exiting nerve root or nerve root ganglion, including compression (decreased diameter) or swelling (increased diameter), compared to the contralateral one. Third, the presence or absence of a displacement of the ipsilateral nerve root by the herniated disc material was also evaluated and compared with the contralateral one. The above assessments were performed on the axial T1- and T2-weighted images.

\section{Data analysis}

The reliability of the MRI evaluation was estimated using the agreement percentage and $\boldsymbol{\kappa}$ statistics for each observer (intraobserver reliability) and among the observers (interobserver reliability). The interpretations were performed in accordance with the consensus of all authors. A value of 1 indicated absolute agreement, whereas a value of 0 indicated agreement no better than chance. The agreement was rated as follows: Poor, $\kappa=0$ to 0.2 ; fair, $\kappa=0.21$ to 0.4 ; moderate, $\kappa=0.41$ to 0.6 ; substantial $\kappa=0.61$ to 0.8 ; and excellent, $\kappa>0.81$. The intraobserver and interobserver agreement

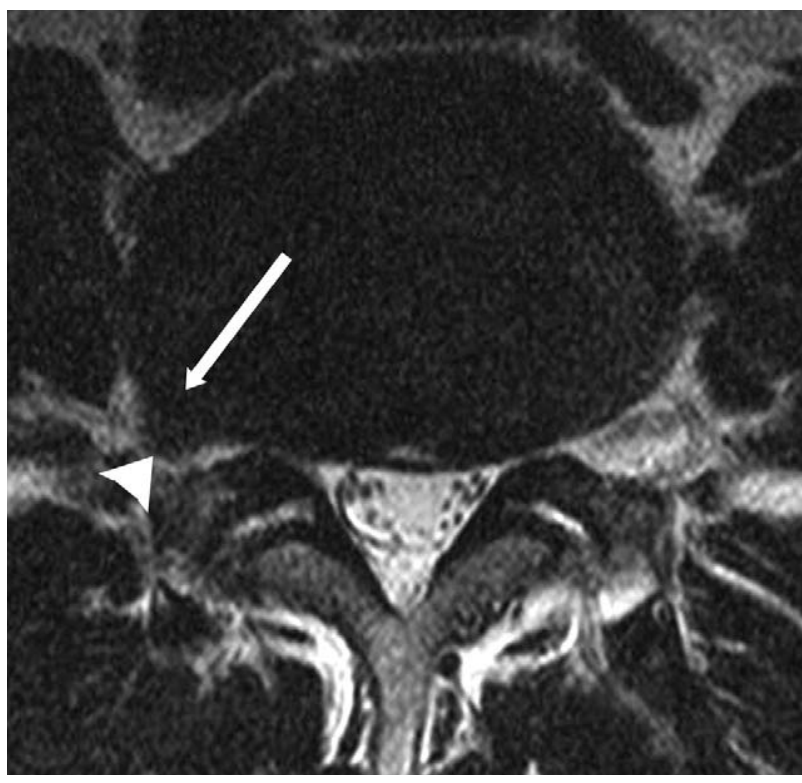

Fig. 3. Fifty-five-year-old man with right extraforaminal disc herniation at the L4-5 level. Subtle asymmetry of the disc contour (arrow) is noted on the axial T2-weighted image. The right exiting L4 nerve root (arrowhead) is swollen compared to the contralateral one.

were calculated using SPSS (ver. 13.0; SPSS Inc., Chicago, IL, USA).

\section{Results}

Of the 60 subjects enrolled in this study, 30 patients had a symptomatic extraforaminal disc herniation. The mean ages of the 30 healthy volunteers (15 males and 15 females) and 30 patients (16 males and 14 females) with symptomatic extraforaminal disc herniation were $40.5 \pm 9.8$ and $38.0 \pm$ 9.2, respectively. The consensus revealed focal eccentricity of disc in 33 cases (55\%, 28 patients and 5 controls), a change in the diameter of the nerve root in 31 cases $(51.7 \%$, 26 patients and 5 controls), and a displacement of the nerve root in 23 cases $(38.3 \%, 19$ patients and 4 controls).

The overall agreement of the three reviewers in determining the presence or absence of a symptomatic extraforaminal disc herniation was $89.4 \%$ (161/180; range 86.7 91.7\%, sensitivity; $90.0 \%$, specificity; $88.9 \%$, positive predictive value; $89.0 \%$, negative predictive value; $89.9 \%$ ). An assessment of paired intraobserver and interobserver reliability revealed a mean Kappa statistic of 0.822 (95\% CI, 0.739 0.905 ; SE, 0.042) and 0.667 (95\% CI, 0.558 0.775; SE, $0.056)$, respectively.

The agreements between the three reviewers in determin- 
ing the presence or absence of focal eccentricity of the disc, changes in the diameter of the nerve root and a displacement of the nerve root were $83.9 \%$ (151/180; range, 83.3 $85.0 \%$ ), $83.3 \%$ (150/180; range, $80.0 \sim 88.3 \%$ ), and $74.4 \%$ (134/180; range, 60.0 81.7\%), respectively.

An assessment of paired intraobserver reliability revealed a mean Kappa statistic of 0.833 (95\% CI, 0.753 0.914; SE, 0.041 ) for focal eccentricity of the disc, 0.656 (95\% CI, $0.545 \sim 0.766$; SE, 0.056) for a change in the diameter of the nerve root, and 0.669 (95\% CI, 0.557 0.782; SE, 0.057) for a displacement of the nerve root. When all MRI findings were taken into consideration, the paired intraobserver reliability had a mean Kappa statistic of 0.724 (95\% CI, 0.666 0.783; SE, 0.030).

An assessment of the paired interobserver reliability revealed a mean Kappa statistic of 0.667 (95\% CI, 0.558 0.775; SE, 0.056) for focal eccentricity of the disc, 0.556 (95\% CI, 0.434 0.677; SE, 0.062) for a change in the diameter of the nerve root, and 0.020 (95\% CI, -0.137-0.176; SE, 0.080 ) for a displacement of the nerve root. When all MRI findings were taken into consideration, the paired interobserver reliability had a mean Kappa statistic of 0.433 (95\% CI, 0.357 0.509; SE, 0.039).

\section{Discussion}

Although obvious focal disc herniation into the foraminal or extraforaminal zone can be seen on MRI, the condition is often overlooked or interpreted as asymmetric or simple disc bulging when the eccentricity of the disc contour is indistinct or subtle.

A failure to recognize the presence of extraforaminal disc herniation with or without foraminal herniation is often responsible for a poor outcome and persistent sciatica, even after surgery ${ }^{10-12}$. Therefore, extraforaminal disc herniation with or without foraminal herniation is an important pathological entity, and a precise diagnosis before surgery is essential.

Extraforaminal lumbar disc herniations beneath or lateral to the facet joint occurs in $2.6 \%$ to $11.7 \%$ of all lumbar disc herniations ${ }^{1,5,13-17}$. However, detection of these herniations is generally difficult. Osborn et al. ${ }^{8}$ reported that one-third of far-lateral herniations have an initial misdiagnosis. In general, most radiologists and clinicians can easily detect posterolateral disc herniations and rarely make a misdiagnosis on spine MRI. However, a diagnosis of the extraforaminal disc herniation can often be overlooked. This condition has remained elusive because of its atypical clinical presentation and inconsistent radiographic findings ${ }^{12}$. To our knowledge, there are no reports on the characteristics and reliable MR findings of extraforaminal disc herniation. Therefore, reliable MRI findings to detect symptomatic extraforaminal disc herniation were assessed.

When nerve root involvement in the extraforaminal zones is suspected, a comprehensive evaluation of the clinical findings, $\mathrm{CT}$ and MRI findings as well as a selective nerve root infiltration and block are necessary to avoid a misdiagnosis 9 . Although selective nerve root infiltration and radiculography are useful diagnostic tools for identifying the precise site and degree of neural compression, this method is invasive and it is difficult to examine several levels simultaneously ${ }^{2}$. Extraforaminal disc herniations are usually not detected on myelography and are often overlooked on the high-resolution CT or MR images ${ }^{12}$. The CT features of extraforaminal disc herniation are nonspecific ${ }^{14,18,19}$. Recently, a detailed evaluation of disc herniation using only CT imaging was determined to be unsuitable, and MR is preferred because it is noninvasive, has high soft-tissue resolution, and multiplanar imaging capabilities ${ }^{20}$. Therefore, MRI may be the preferred method for evaluating a symptomatic patient with both central and lateral spinal canal pathology.

Grenier et al. ${ }^{3}$ concluded that MRI is useful for analyzing extraforaminal disc herniation. They added a $15 \sim 30^{\circ}$ angled frontal view oblique caudally and anteriorly, following the course of the nerve roots. However, this image is not generally included in routine spine MRI protocols. This study evaluated all extraforaminal disc herniations on general axial T1- and T2-weighted images. Jenis and $\mathrm{An}^{10}$ suggested that the use of parasagittal images allows visualization of the foramina along the length of the lumbar spine while allowing superior resolution of the associated disc and vertebral body changes. However, the extraforaminal herniations could not be confirmed on the sagittal images. Purely extrafominal disc herniation could not be visualized on the sagittal images because sagittal scanning of spine MRI at our institution was obtained within the range of the vertebral body width. However, the disc contour or nerve root on the axial images at the same level were assessed more carefully when disc herniation into the neural foramen was partially observed on the sagittal plane in the case of mixed type (extraforaminal with foraminal) herniations.

This study showed that the overall agreement in determining the presence or absence of symptomatic extraforam- 
inal disc herniation using the MRI findings, including focal eccentricity of the disc, changes in the diameter of the nerve root, and a displacement of the nerve root, was $89.4 \%$ (161/180; range, 86.7 91.7\%). The assessment of the paired intraobserver and interobserver reliability in determining the presence or absence of a symptomatic extraforaminal disc herniation revealed a mean Kappa statistic of excellent and substantial, respectively. The assessment of the paired intraobserver and interobserver reliability with each MRI finding revealed a mean Kappa statistic of excellent and substantial for focal eccentricity of the disc, substantial and moderate for a change in the diameter of the nerve root, and substantial and poor for a displacement of the nerve root, respectively. This study showed that the focal eccentricity of the disc was the most reliable MRI finding.

There were some limitations in our study. First, the number of patients included in this study was small. Second, other imaging protocols were not added for a detailed evaluation of extraforaminal disc herniation because this study was retrospective.

\section{Conclusions}

There are three possible MRI findings in determining the presence or absence of symptomatic extraforaminal disc herniation. Among these MRI findings, focal eccentricity of disc was found to be the most reliable. However, it is important to recognize that even with these MRI findings, it is possible to misdiagnose symptomatic extraforaminal disc herniation

\section{REFERENCES}

1. Abdullah AF, Ditto EW 3rd, Byrd EB, Williams R: Extreme-lateral lumbar disc herniations: clinical syndrome and special problems of diagnosis. J Neurosurg 1974; 41: 229-234.

2. Baba H, Maezawa Y, Furusawa N, et al: Extraforaminal lumbar disc herniation at two contiguous intervertebral levels. Spinal Cord 1997; 35: 725-728.

3. Grenier N, Greselle JF, Douws C, et al: MR imaging of foraminal and extraforaminal lumbar disk herniations. J Comput Assist Tomogr 1990; 14: 243-249.

4. Wiltse LL, Spencer CW: New uses and refinements of the paraspinal approach to the lumbar spine. Spine (Phila $\mathrm{Pa}$ 1976) 1988 ; $13:$ 696-706.
5. Abdullah AF, Wolber PG, Warfield JR, Gunadi IK: Surgical management of extreme lateral lumbar disc herniations: review of 138 cases. Neurosurgery 1988; 22: 648-653.

6. Fankhauser H, de Tribolet N: Extreme lateral lumbar disc herniation. Br J Neurosurg 1987; 1: 111-129.

7. Hood RS: Far lateral lumbar disc herniations. Neurosurg Clin N Am 1993; 4: 117-124.

8. Osborn AG, Hood RS, Sherry RG, Smoker WR, Harnsberger HR: CT/MR spectrum of far lateral and anterior lumbosacral disk herniations. AJNR Am J Neuroradiol 1988; 9: 775-778.

9. Kunogi J, Hasue M: Diagnosis and operative treatment of intraforaminal and extraforaminal nerve root compression. Spine (Phila Pa 1976) 1991; 16: 1312-1320.

10. Jenis LG, An HS: Spine update: lumbar foraminal stenosis. Spine (Phila Pa 1976) 2000; 25: 389-394.

11. O'Hara LJ, Marshall RW: Far lateral lumbar disc herniation: the key to the intertransverse approach. J Bone Joint Surg Br 1997; 79: 943-947.

12. Maroon JC, Kopitnik TA, Schulhof LA, Abla A, Wilberger JE: Diagnosis and microsurgical approach to far-lateral disc herniation in the lumbar spine. J Neurosurg 1990; 72: 378-382.

13. Kurobane Y, Takahashi T, Tajima T, et al: Extraforaminal disc herniation. Spine (Phila Pa 1976) 1986; 11: 260-268.

14. Gado M, Patel J, Hodges FJ 3rd: Lateral disk herniation into the lumbar intervertebral foramen: differential diagnosis. AJNR Am J Neuroradiol 1983; 4: 598-600.

15. Kornberg M: Extreme lateral lumbar disc herniations: clinical syndrome and computed tomography recognition. Spine (Phila Pa 1976) 1987; 12: 586-589.

16. Leonardi M, Biasizzo E, Fabris G, Penco T, Bertolissi D: CT evaluation of the lumbosacral spine. AJNR Am J Neuroradiol 1983; 4: 846-847.

17. Macnab I: Negative disc exploration: an analysis of the causes of nerve-root involvement in sixty-eight patients. J Bone Joint Surg Am 1971; 53: 891-903.

18. Eckardt JJ, Kaplan DD, Batzdorf U, Dawson EG: Extraforaminal disc herniation simulating a retroperitoneal neoplasm. Case report. J Bone Joint Surg Am 1985; 67: 1275-1277.

19. Schubiger O, Valavanis A, Hollmann J: Computed tomography of the intervertebral foramen. Neuroradiology 1984; 26: 439-444.

20. Ozturk C, Tezer M, Sirvanci M, et al: Far lateral thoracic disc herniation presenting with flank pain. Spine J 2006; 6: 201-203. 